\title{
The Implicit Definition of the Set-Concept
}

\author{
F.A. Muller \\ Utrecht University
}

\begin{abstract}
Summary
Once Hilbert asserted that the axioms of a theory 'define' the primitive concepts of its language 'implicitly'. Thus when someone inquires about the meaning of the set-concept, the standard response reads that axiomatic set-theory defines it implicitly and that is the end of it. But can we explain this assertion in a manner that meets minimum standards of philosophical scrutiny? Is I. Jané [2001] wrong when he says that implicit definability is "an obscure notion"? Does an explanation of it presuppose any particular view on meaning? Is it not a scandal of the philosophy of mathematics that no answers to these questions are around? We submit affirmative answers to all questions. We argue that a Wittgensteinian conception of meaning looms large beneath Hilbert's conception of implicit definability. Within the specific framework of P. Horwich's recent Wittgensteinian theory of meaning called semantic deflationism, we explain an explicit conception of implicit definability, and then go on to argue that, indeed, set-theory, defines the set-concept implicitly according to this conception. We also defend Horwich's conception against a recent objection from the Neo-Fregeans B. Hale and C. Wright [2001]. Further, we employ the philosophical resources gathered to dissolve all traditional worries about the coherence of the set-concept, raised by Frege, Russell and Max Black, and which recently have been defended vigorously by M.F. Hallett in his magisterial monograph Cantorian set-theory and limitation of size [1984]. Until this day, scandalously, these worries have been ignored too by philosophers of mathematics.
\end{abstract}





\section{Contents}

1 Introduction $\quad 1$

2 The Rise of Multitarian Wholism 3

2.1 The Whole Set . . . . . . . . . . . . . . . 3

2.2 The Multitarian Set . . . . . . . . . . . . . . 5

2.3 The Pure Set . . . . . . . . . . . . . . . 7

2.4 From the Fire into the Flames . . . . . . . . . . . . . . 7

2.5 Verbal Juggling Acts . . . . . . . . . . . . . . . . . 8

3 The Sources of Perplexity $r$

4 Dense Thickets of Mystery $\quad 11$

5 Implicit Definability $\quad 15$

6 Semantic Deflationism 18

7 Implicit Definability Revisited 21

8 Attack of the Neo-Fregeans 24

9 The Fall of Multitarian Wholism 26

$\begin{array}{ll}10 \text { Exitum } & 30\end{array}$

$\begin{array}{ll}\text { References } & 32\end{array}$ 



\section{Introduction}

Axiomatic set-theory is a theory about sets and nothing but sets. The importance and fertility of axiomatic set-theory for mathematics, logic, the philosophy of mathematics and metamathematics, e.g. model-theory, does not stand in need of emphasis. ${ }^{1}$

But what is a set? What does the word 'set' mean? How do we have to understand this pivotal concept of mathematics? These queries may sound tantalisingly awkward in the light of the success of axiomatic set-theory. Are we dealing here with a desperate philosopher of mathematics in search of a subject? Well, these queries are prompted by specific worries concerning the clarity and coherence of the set-concept, raised by the likes of Frege, Russell, Weyl, Skolem and Max Black. We can hardly consider these to be desperate minds in search of a subject. I believe their specific worries merit attention, in particular because these worries are not dead and buried in past history. In his magisterial monograph on Cantorian set-theory, M.F. Hallett [1984: 299-301] has endorsed them vigorously by upholding that "two dense thickets of mystery" surround the set-concept, and that axiomatic set-theory fails to achieve "conceptual clarity", which "was genuinely part of Cantor's aim in pursuing set-theory." One may bark at these worries about the meaning of the set-concept. But philosophers should do better than bark back. Let us leave the barking to the dogs.

I submit it is a bit of a scandal that so far analytic philosophers have not brought their apparatus of analysis to bear on the practice of set-theory — but instead have restricted themselves to conducting debates that have little if any relevance to the practice of mathematics. Let me not be misunderstood. I do not say these debates should not be conducted or are uninteresting. Far from it. Nor do I say that set-theoreticians are somehow intellectually ill and need to be cured by philosophical therapy. Far from it. But what I also say is that the practice of set-theory raises certain questions about that practice which philosophers should consider to be meaningful and open to philosophical exploration. One such question is how this 'implicit definability' works; another is why the implicit definition should dissolve the worries concerning the clarity and coherence of the set-concept (one of Hallett's "dense thickets of mystery"); and yet another question is how this dissolution precisely works.

In the next Section (Section 2), we attempt to state the worries about the clarity and coherence of the set-concept as convincingly as we can. The dissolution of these worries begins with some detailed comparisons of how various crucial expressions involving the setconcept are used differently in different contexts (Section 3); these comparisons are made against the background of a Wittgensteinian view of meaning, which takes the use of ex-

\footnotetext{
${ }^{1}$ See Fraenkel et al. [1973], the tomes Hodges [1993] and Kanamori [2003] and references therein. When we write 'set-theory' in the present paper we mean the relevant branch of mathematics. By 'axiomatic set-theory' we mean any well-axiomatised set-theory, notably standard 'Zermelo-Fraenkel set-theory' (ZFC), which is formulated in the usual, extremely simple formal language (denoted by $\mathcal{L}_{\epsilon}$ ). We frequently omit the adjective 'axiomatic' when the context allows it. For those who have forgotten: the extreme simplicity of $\mathcal{L}_{\epsilon}$ resides in the fact that it is 1st-order, that it has a single type of primitive term (set-variables) and a single primitive predicate (the binary membership-relation $\in$ ).
} 
pressions to constitute their meaning. ${ }^{2}$ The next step is to enter the road of making sense of Hilbert's assertion that the axioms of set-theory define the set-concept and the membershiprelation implicitly. ${ }^{3}$ For how exactly does this work: by asserting a word is implicitly defined by a number of hand-picked sentences in which the word occurs it suddenly obtains a meaning? Must we take such acts of semantic magic for granted? Is being implicitly defined by a theory "an obscure notion", as Jané [2001: 148] recently has remarked in passing, and beyond rational salvation? We relate the step on the mentioned road of making sense of implicit definition to Hallett's explicit criticisms of the set-concept and argue that his specific criticism is, by our lights, ill-founded (Section 4). Since the notion of an implicit definition has been criticised rather severely, the road to making sense of it has some wellknown obstacles, e.g. A.N. Prior's 'runabout inference-ticket' [1960]. In Section 5, we explain four Problems that face any account of implicit definability, some of which are raised by the worries treated in the previous Sections. In Section 6, we briefly outline the salient parts of Horwich's Wittgensteinian theory of meaning, called semantic deflationism; within its framework we make an attempt to solve the four Problems (discussed in Section 5). Subsequently, in Section 7, we present and defend our revision of Horwich's notion of implicit definability and argue that it solves all four Problems. Recently the Neo-Fregeans B. Hale and C. Wright [2001] have levelled "a decisive objection" against Horwich's notion; in Section 8, we argue that their objection is incoherent. In Section 9, we argue that the axioms of ZFC qualify as an implicit definition of the set-concept and the membership-relation; we also show in detail how the dissolution of the worries raised in Section 2 works. Finally, in Section 10, we point out that our analysis ought to count as 'the eighth' argument in favour of semantic deflationism - salute Horwich.

Besides the first class of worries challenging the clarity and coherence of the set-concept 'itself' (comprising both of Hallett's "dense thickets of mystery"), there is a second class of worries generated by certain well-known meta-mathematical theorems about set-theory (the Löwenheim-Skolem theorems and the various undecidability theorems): they challenge the adequacy of axiomatic set-theory to capture the set-concept unambiguously and precisely. Although not entirely unrelated to the worries of this paper, these meta-mathematically grounded worries are not addressed in Hallett [1984]; we do not address them either hic et nunc, but we do so with full force in a separate paper complementary to the present one (in Muller [2004]).

\footnotetext{
${ }^{2}$ We are notably not concerned here with Wittgenstein's finitistic-like philosophy of mathematics.

${ }^{3}$ The idea of an implicit definition, also called a 'contextual definition', is due to Frege (who eventually came to reject it); both Hilbert and Poincaré embraced it. The term should not be confused with E.W. Beth's formal model-theoretic notion of implicit definability, which concerns primitive predicates in a 1st-order language.
} 


\section{The Rise of Multitarian Wholism}

\subsection{The Whole Set}

Suppose there are a number of tomatoes on the table. Suppose we are asked to verify the assertion there are seventeen tomatoes on the table. We know how to do this: we count them. What? Sixteen? We count them again. Sixteen. We conclude the assertion was false: there are sixteen tomatoes on the table, not seventeen.

Suppose next we are asked to verify the assertion that the tomatoes on the table form a set. What are we supposed to do now? We turn for help to the founding father of settheory. Here comes Cantor's inevitable description of a set from Beiträge zur Begründung der transfiniten Mengenlehre (Cantor [1895, §1]):

A set is the result of combining into a whole definite, distinct objects of our intuition or thought - which are called the members of the set.

So this is what we have to do with the tomatoes according to Cantor: we have to combine them into a whole, into a single object. Well, how does one combine tomatoes into a whole? How does one turn them into a single object? ${ }^{4}$

We can prima facie think of two things to do: (i) group the tomatoes together, make a heap of them, and perhaps put them in the paper bag that happens to lie near the table; or (ii) inspect each of the sixteen things carefully in order to verify they meet the monadic predicate Tomato(.), defined as a conjunction of a number of predicates 'directly' verifiable by our senses, concerning colour, softness, shape and taste. (We want to mention that this last-mentioned, class-rendition of set, i.e. as the extension of a predicate, was at best only occasionally adduced by Cantor. ${ }^{5}$ )

Before we examine (i) and (ii) more closely, let us quickly remove one objection against the validity of the question of what we are supposed to do when asked to verify whether the tomatoes form a set. The objection is that the set of sixteen tomatoes is an abstract, perhaps mental object, rather than a concrete, physical object; it is misguided to display perplexity about what we must do physically in order to arrive at an abstract object. This objection does not hold water in the light of how we began: the natural number 17 also is an abstract object but it was perfectly clear what we had to do when asked to verify the assertion there are

\footnotetext{
${ }^{4}$ Black [1971: 618] essentially raised precisely this question.

${ }^{5}$ For example, Cantor $[1895, \S 1]$ speaks of “... jedes Viele, welches sich als Eines denken lässt, d.h. jeden Inbegriff bestimmter Elemente, welcher durch ein Gesetz [charitably read: a set-theoretical predicate, FAM] zu einem Ganzen verbunden werden kann." Nonetheless Cantor honored the distinction between the combinatorial set and the logical class, as for example in his criticism of Frege; cf. Hallett [1984: 45-48, 126-128]. Lavine [1994: 85-86] denies that "ein Gesetz" must be identified with a set-theoretical predicate; it must be identified with the generation principles of the ordinal numbers, as the context of Cantor's quotation supports according to Lavine. Although this would rule out (ii) as an interpretation of Cantor's quotation, (ii) stands tall as a possible interpretation of what exactly to do when asked to verify whether the tomatoes on the table form a set. No matter what Cantor's intentions were, we want to investigate this possible interpretation (ii) here.
} 
seventeen tomatoes on the table: we simply counted them. A three year old child can do it and no amount of philosophical argumentation can do away with this fact. Such a child will admittedly point to the tomatoes one by one with its finger, or will pick up the tomatoes in its hands and will move them around, or will count them out loud, but none of all this is necessary to obtain the correct answer - we, adults, can do it without lifting a finger or making a noise. Furthermore, the abstract object of a set is just as applicable to the concrete world as is the abstract object of a natural number. The expression 'a set of tomatoes' ought to be as meaningful as the expression 'sixteen tomatoes'.

Having now removed the prima facie objection against the validity of the question, we turn to our two possible interpretations of this question. In so far as we can claim to grasp the set-concept, both interpretations (i) and (ii) of what we have to do in order to form a set of sixteen tomatoes are inadequate. We explain.

(i) In spite of the fact that Cantor's symbolic notation with curly brackets, as in $2=\{0,1\}$ (0 and 1 seem imprisoned), sits comfortably next to assertions of spatial confinement (think of the grouping together of the tomatoes and putting them into a paper bag), the set-concept is not supposed to rely on any spatial notions. Yet spatial notions are manifestly present in interpretation (i); it relies on them. The 'whole' (the object) that a set is supposed to be is not a volume in Euclidean space or some area in the Euclidean plane, illustrations of the basic set-theoretic operations with Venn-diagrams notwithstanding. Set-theory is not Geometry. And we never suppose that sets are paper bags; as we pointed out before, sets are not concrete objects. If it is replied that of course a set is not a paper bag but it is supposed to be some non-spatial, abstract analogue of a paper bag, we display perplexity: we simply do not understand the phrase 'some non-spatial, abstract analogue of a paper bag' anymore as we understand, for instance, the phrase 'some non-temporal, abstract analogue of a year'. We even feel inclined to say that anyone who is making such assertions is uttering nonsense.

(ii) Also the verification that each of the sixteen things on the table meets the predicate Tomato(.) does not help. What such a verification achieves - when completed - is that we are certain we are dealing with sixteen genuine tomatoes. How is the abstract object of the extension of the predicate (a class ${ }^{6}$ ) conjured up after we have verified that Tomato(.) holds for every single object on the table? After inspection we are left with 16 inspected tomatoes on the table. Where does the class, then, come from?

The abstract object of a class is as perplexing as the abstract object of a set. But for the set-concept - again in so far as we can claim to grasp it - , meeting the predicate Tomato(·) for its members is immaterial. For suppose one tomato is an artificial one. We are then still supposed to be able to make a set of fifteen real tomatoes and one plastic one. (One can come up with a different, weaker predicate; then we add a carrot; someone comes up with the predicate RealOrArtificialVeg(.); then we add a beetle; \&c.) Further, axiomatic settheory provides ample testimony for the fact that we can have a set without a predicate. The Axiom of Choice, for instance, notoriously asserts the existence of a set without giving

\footnotetext{
${ }^{6}$ Whitehead \& Russell [1910: 72]: “... an extension (which is the same as a class) ...".
} 
a predicate for it. Furthermore, if having a predicate were necessary for the existence, or the determination, of a set, there could be no more than $\aleph_{0}$ sets in the domain of discourse of any set-theory formulated in $\mathcal{L}_{\epsilon}$ because there are no more than $\aleph_{0}$ names available in its language $\mathcal{L}_{\epsilon}$. But in any set-theory with an axiom of infinity there are demonstrably unimaginably more sets available.

We conclude that neither (i) spatial notions nor (ii) the presence of a predicate are essential to the set-concept.

But when we ignore the fact that we are dealing with genuine tomatoes and their spatial arrangements - both being mere pedagogical crutches, artifacts of a particular illustration, inessential for the set-concept - , the example of the tomatoes we have been focusing on evaporates: if we 'abstract from' the tomatoes, from their properties as codified in Tomato(.) and from their spatial arrangements, what are we left with? An empty table it seems.

Or perhaps sixteen "lauter Einsen" (Cantor)? That is, sixteen indistinguishable yet distinct Cantorian "definite distinct objects of thought"? But granting Cantor the troublesome notion of 'indistinguishable yet distinct definite objects of thought', what we are left with then is, according to Cantor [1895: 481-482], the cardinality of the set, whereas we are still after the set itself and the set itself comes logically prior to its cardinal number.

We conclude that our perplexity about the seemingly so simple assertion that the sixteen tomatoes on the table form a set, in particular about Cantor's description they are "combined into a whole", has not been resolved. The worry stands tall.

\subsection{The Multitarian Set}

The most mysterious yet undoubtedly essential feature of a set is that it is, according to Cantor, "a many thought of as one", a multitude as a single object (see the quotation in endnote 5) — notice that the laws of English grammar have to be broken to express it. Black [1971: 616] compared Cantor's idea of "a many as one" to the Catholic idea of the Holy Trinity, which is 'three as one': the Father, the Son and the Holy Spirit are to be conceived as one in God. Black concluded that the set-concept is theological rather than mathematical in nature (ibid.). Whitehead \& Russell argue in a footnote of Principia Mathematica as follows:

If there is such an object as a class, it must be in some sense one object. Yet it is only of classes that many can be predicated. Hence if we admit classes as objects, we must suppose that the same object can be both one and many, which is absurd. ${ }^{7}$

Indeed, the directive to think of 'a many' as one object looks alarmingly much like the directive to think of a circle as rectangular, of black as white, of a gluon as a fermion, \&c.

What if we consider a set with a single member? What sense does it make to say: 'to combine a single tomato into a whole, into another single object'? We can sensibly assert

\footnotetext{
${ }^{7}$ Whitehead \& Russell [1910: 72]. For this reason, they reject classes, but still use them because classes can always be replaced with 'incomplete symbols'.
} 
this when the tomato is cut into pieces and the pieces are fit together; but that is not the case here. Set-theory is not mereology. What sense does it make to say that the set of a single tomato is 'a many thought of as one object'? Certainly one is not an instance of 'many'. The word 'many' means 'more than one', at the very least.

If it is, then what about the empty set? (Notice the spatial connotations of this name, which are wholly inappropriate in the context of set-theory - memberless set would be more appropriate.) If we first need Cantorian definite, distinct objects of thought (ddots for brevity), in order to combine them into a whole so as to obtain a set, then there is no set whenever there are no ddots. If a set is the kind of thing that has members, then there is no set when there are no members. In commenting on E. Schröder's Vorlesungen über die Algebra der Logic, Frege wrote:

A set, in the sense in which we have so far used the word, consists of objects; it is an aggregate, a collective unity of them; if so, it must vanish when these objects vanish. If we burn down all the trees of a forest, we thereby burn down the wood. Thus there can be no empty set. ${ }^{8}$

The idea of an empty set is even more stultifying than the idea of a singleton set, for it is 'no thing seen as one'. Surely zero is not an instance of 'a many'. For reasons similar to the ones presented above, Black [1971] condemned Cantor's descriptions as "mystification on stilts."

It may look silly to bring in the extremely uninteresting empty set, but we should bear in mind that the cumulative hierarchy of all pure sets in ZFC begins with exactly one set: $\varnothing$. On Cantor's account there can be no empty set, but its existence is the conditio sine qua non for the domain of discourse of pure set-theory.

We can summarise these criticisms by saying that the 'whole-worry' and the 'manyworry' are two ways of criticising that a set of objects can itself be considered as an object: the whole-worry inquires about the nature of this object, the many-worry inquires about how this single object can stand for many (other) objects. We formulate this worry as a thesis:

Multitarian Wholism. No clear and coherent meaning can be attached to the Expressions 'to combine into a whole' and 'to see many as a single object'; therefore the set-concept has no clear and coherent meaning either.

Multitarian wholism is not yet the end of it.

\footnotetext{
${ }^{8}$ In Frege [1984: 212]. For evident reasons I have replaced 'class' with 'set', because Frege adopts, for the purpose of his review, Schröder's description of a 'domain', which is the same as Cantor's description of a set (p. 3); and because Frege announces to use the word 'class' synonymously to 'domain': "Instead of 'domains' we may here always also say 'classes', if we take classes to be collective wholes, such as for example a wood, and do not bring them into connexion with concepts," (ibid., 210-211, for that Frege reserves the word "extension"); ergo, for Frege, 'classes' are not extensions of predicates, as today is the standard terminology, but they are Cantorian sets.
} 


\subsection{The Pure Set}

Let us now suppose that we somehow fully understand the 'trinitarian whole', or better multitarian whole, that a set is supposed to be; in other words, we suppose that Multitarian Wholism is false. We know what it means to combine sixteen tomatoes into a whole and to think of a many as one object. Next we want to understand the concept of a pure set. If it is replied that we only have to replace the sixteen tomatoes with sixteen sets, we are destined to run around in circles whenever these sixteen sets are also required to be pure (as they must be), because we first need to have some understanding of the things being combined into a whole before we can even begin to consider this whole. We can replace the tomatoes with tables or tommies, but not with pure sets, because pure sets are exactly the things we wanted to understand in the first place.

Furthermore, in order to formulate the Axiom of Extensionality we need to have an identity criterion for the members of a set, because Extensionality asserts that any two given sets are identical if their members are identical. But in order to know when two pure sets are identical, we arguably first need to know what a pure set is, because the members of two pure sets whose identity we want and must inquire into are by mathematical necessity all pure sets themselves. How can we inquire meaningfully into the conditions that two pure sets have to satisfy in order to pronounce them identical if we have no clue of what their members (also pure sets) are? We must understand what a pure set is in order to understand what a pure set is. Conceptually we find ourselves in an uncomfortable circle.

\subsection{From the Fire into the Flames}

Hallett [1984: 300-301] brought to the fore that the concept of a natural number, a real number, a function between real numbers, a relation, a point in space, a figure in the plane, and other concepts of classical mathematics have never evoked the perplexity that the multitarian whole evokes. We cannot claim, for instance, that the presence and recognition of borderline cases of functions, as perceived by XIXth-century mathematicians (Dirichlet's function, Weierstrass' function), evoke similar perplexities. Hallett's observation is that the reduction of all concepts of mathematics to the set-concept does not lead to an accompanying gain in clarity. One of the aims of analysing 'complex' concepts into 'simpler' constituents is that such an analysis must, whenever successful, enhance our understanding of the 'complex' concepts; in other words: such analyses must lead to explications. This we would consider a philosophical virtue of the process of analysis. Without such virtues, what would be the point of analysis in the first place? Why would we reduce every single concept of mathematics to the set-concept if this obscures rather than illuminates these concepts? The success of axiomatic set-theory, in particular as a founding theory of mathematics, makes the absence of such virtues embarrassingly visible. The Herculean ontological achievement of set-theoretical reductions is not matched by a parallel achievement in the increase of clar- 
ity. On the contrary, from the considerations above it seems we suffer from heavy losses in clarity. Hallett concludes that the ontological gain is annihilated by an epistemological loss [1984: 301].

Of course, at the end of the day we may (and plausibly should) judge the ontological achievement to outweigh these epistemic oddities. But even then the fact remains they are epistemic oddities. We are concerned with them and try to dissolve their causes without overtly appealing to ontological achievements or to any other balancing techniques from the Department of Accountancy. Also without Herculean ontological achievements there should be clear and coherent meaning.

\subsection{Verbal Juggling Acts}

From our brief analysis of Cantor's descriptions of a set as "ddots combined into a whole", occasionally "by means of a law", and "a many which can be thought of as one", we conclude that Cantor's tacit claim (if he had it) that these descriptions are explicatory is illgrounded. As we have seen, the terms used in these descriptions stand as much in need of elucidation as the concept they are supposed to elucidate. Cantor's descriptions seem a kind of, sit venia verbo, verbal juggling act. After some more verbal juggling with words like 'collection', 'assembly', 'aggregate', 'group', 'multiplicity', 'elements', 'to gather', 'to combine into a whole', 'to think of as one object' and what have you, we soon reach the point where we suspect that in this fashion we shall keep on juggling until we are paralysed. There simply seem to be no concepts available that qualify for occurring in the description of a set such that we unhesitatingly accept the resulting description as an explication. The inevitable conclusion seems that looking for them is pursuing a will-o'-the-wisp.

In order to come to terms with this misguided pursuit, we first have to understand why we (and Cantor) embarked on this explicatory pursuit in the first place. In other words, we have to put the finger on exactly where our perplexity originates.

\section{The Sources of Perplexity}

Our perplexity originates precisely here: the expressions used by Cantor in his descriptions of the set-concept are used non-standardly. We claim that precisely here begins the road that will lead to the dissolution of our perplexity. We explain this shortly; first a word on Wittgenstein's view of meaning.

Wittgenstein famously held the use of an expression to be the clue to its meaning [1979: 48]:

I have suggested substituting for 'meaning of a word', 'use of a word', because use of a word comprises a large part of what is meant by 'the meaning of a word'. Understanding 
a word will thus come to knowing its use, its applications. The use of a word is what is defined by the rules, just as the use of the king of chess is defined by the rules. And just as the shape and material of the king of chess are irrelevant to its use, so are the shape and sound of a word to its use.

To know the meaning of an expression is to understand it; and to understand an expression is to possess the capability to use it correctly, i.e. in agreement with the rules of the semantic grammar that can be considered to govern the use in a particular linguistic practice, or context, or in Wittgensteinian slang: to govern the language-game we happen to play. These semantic rules fix the semantic grammar of words and expressions, just as syntactic rules fix the syntactic grammar of sentences in a language (their grammatical form). Unlike syntactical rules, however, which are transcontextual, i.e. they generally hold in every context, semantic rules for a given expression can be contextual, i.e. for the same expression they can vary from one context to another. So, succinctly, an expression can acquire meaning iff it is possible to have some context and a language in which the expression occurs and can be used successfully by a community of language-users. In principle every expression can acquire a meaning, although, of course, not every expression has a meaning.

Suppose some art critic asserts we have to look at Dimanche d'été à la Grande Jatte not by focusing on the individual colour-dots, but as a whole, to see it as one, after we have taken a few steps back from the painting. We understand that. Suppose some music critic tells us we have to listen to Einstein on the beach not as a sequence of individual notes played by separate instruments and of separate words uttered by voices, but we have to experience it as a whole, to listen to it as one piece of music. We understand that; it actually takes a sustained effort not to do so. Suppose some literary critic says we have to read Molly Bloom's interior monologue not by spelling every word and pause to ponder its meaning, but we have to undergo the flowing monologue as a whole, as one verbal stream of consciousness. We understand that. And so forth. Whatever the expressions 'as a whole' and 'as one' mean in these examples, and whatever their finer differences in these examples are, they surely are not synonymous to the same expressions in 'to combine sixteen tomatoes into a whole' and 'to see the tomatoes as one object'. For instance, when we are told to consider the set of colour-dots that constitute Dimanche d'été à la Grande Jatte, we are supposed to do something entirely different from drowning delightfully in Seurat's painting, which is exactly what the art critic invites us to do. When we are told to consider the set of played notes and sung words that constitute Einstein on the beach, we are supposed to do something different than listening to Philip Glass' remarkable piece of minimal music. When we are told to consider the set of words that form Molly Bloom's interior monologue, we are supposed to do something different from reading the final Chapter of Ulysses. The expression 'to see as one object' is used non-standardly by Cantor according to the standards of use which obtain in the contexts mentioned above.

Also the expression 'to combine' is used by Cantor non-standardly, in the following sense. An astronomer says he has combined all available observations in a model for the behaviour of a certain galaxy; a carpenter says to have combined the pieces of wood into a whole; the 
arithmetician says to have combined the given numbers to obtain this number; the general says the army has to combine all its striking power left; the president says we have to combine all our resources to fight this crisis; and so forth. We understand what is meant by 'to combine' in all these different contexts. No conceptual problems arise. No perplexity is in sight. But again, all mentioned expressions including 'to combine' surely are not synonymous with 'to combine sixteen tomatoes into a whole'. In every case one easily discerns differences in meaning. What all examples above have in common, for instance, is that some definite, clear action is supposed to be undertaken, whereas in the case of the sixteen tomatoes we previously arrived at the conclusion that it is a mystery what we are supposed to do with the sixteen tomatoes when asked to combine them (into a whole). It is not clear what action we must undertake with the tomatoes. It seems we carry over the expression 'to combine' from contexts where its meaning is comparatively clear into a context where it becomes obscure.

Now, the fact that Cantor uses the expressions 'to combine into a whole' and 'to think of a many as one' in a manner that deviates from standard use is not something we criticise. What we criticise is Cantor's intention (if he had it) that these expressions are used standardly and are employed to explicate the set-concept, i.e. to describe it in familiar terms used standardly. For surely to explicate a concept in terms of other words used non-standardly is no explication at all: the non-standard use of these other words would stand in as much need of clarification as the concept they are supposed to clarify. If Cantor had clearly stated in advance that his descriptions were never intended as explications of the set-concept in familiar terms used standardly, but as proposals how the novel set-concept is to be used in combination with familiar terms, as a new rule of the semantic grammar of the set-concept, then the perplexity we voiced would never have arisen in the first place. If it had arisen nonetheless, we could explain its rise as a strange refusal to accept a novel concept, irreducible to familiar terms used standardly. The question then would have been whether we either stubbornly want to pamper our perplexity in the face of a proposal to widen our conceptual horizon, or whether we want to overcome our perplexity, want to accept this proposal and, then, let the wonderful combination of reason and imagination take its course.

There are definite resemblances (as well as differences) between the novel set-concept and other familiar concepts used standardly. For example, the expression 'there are in this paper bag sixteen tomatoes' and the set-theoretic sentence 'the Von Neumann ordinal-set 16 has sixteen members', i.e. \#16 = 16 (where \#S stands for the cardinal number of set $S$ ), have to be understood in a very similar sense. When we add three potatoes to the tomatoes and assert to have a paper bag with nineteen pieces of vegetable, this has to be understood in a sense very similar to the sense of the following set-theoretic assertion about ordinals: $\#(16+3)=19$. In fact, at this juncture all pedagogical examples used to introduce the setconcept to pupils of secondary school can be listed: they focus on resemblances in the use of the set-concept and the use of sibling concepts the pupils are much more familiar with - a flock of sheep, a crowd of people, a pack of wolves, a regiment of soldiers, a swarm of bees, a board of directors, a herd of buffaloes, a class of pupils, a deck of playing-cards \&c.

When confronted however with some use of the novel set-concept for which there are no 
resemblances in use available, we are on our own and enter semantic terra incognita.

Well, how do we proceed when we are on our own? How to find our way in terra incognita? Previously we stated our scepticism about 'verbal juggling acts' to obtain an explicatory description of the set-concept. These acts were of no avail. We now possess a clear view on our chase for explicatory willow-o'-the-wisps: if the verbal juggling acts are performed with familiar concepts used standardly, then these acts are irrelevant for our intention to consider the set-concept as a genuinely novel concept. This intention, then, commits us to say how to proceed when we are on our own, i.e. when no resemblances in use are available we are accustomed to. We can find our way in terra incognita only after we have charted it; if no natural infrastructure is present, as is usually the case in unknown territory, we must build it ourselves - no help will come from above.

We now have proceeded farther on the road towards a dissolution of the perplexity of multitarian wholism; in the next Section we continue on this road.

\section{Dense Thickets of Mystery}

A definition of some expression is semantic, it is an expression in the same language as in which the defined expression occurs. The meaning of the definiendum is declared to be the same as the meaning of the definiens; a synonymous expression has been introduced. Definiens and definiendum can be interchanged salve significate; their use is governed by the same rules. This is the standard notion of a definition, as in: set $S$ is ultimate iff it is not a member of any other set; a physical system is closed iff its total energy is conserved over time; \&c. Most expressions in our language, however, do not have explicit definitions. Hilbert and Poincaré regarded axiomatisations as achieving something in between an explicit definition and having no definition, namely having an implicit definition. In the penultimate letter, dated 22 September 1900, of the illuminating exchange between Hilbert and Frege on definitions and axioms, Hilbert summarised his view on the status of axioms as follows:

In my opinion, a concept [primitive notion, FAM] can be fixed logically only by its relations to other concepts. These relations, formulated in certain statements, I call axioms, thus arriving at the view that axioms (perhaps together with propositions assigning names to concepts) are the definitions of the concepts. ${ }^{9}$

In his axiomatisation of Cantorian set-theory, Von Neumann explicitly adhered to the same view:

By 'set' we understand here (in the sense of the axiomatic method) something of which one does not know anything more and does not want to know anything more than what follows from the axioms. ${ }^{10}$

\footnotetext{
${ }^{9}$ Hilbert in Frege [1980: 51], our italics.

${ }^{10}$ Neumann [1925: 36], our translation.
} 
So, succinctly, a notion is implicitly definable iff we can axiomatise it. Here we have a definiendum without a definiens, so that using them interchangeably is no longer possible. Instead we have a variety of rules of how to use the new concept in combination with other concepts.

Now, when we restrict ourselves to mathematics, Wittgenstein's 'social' conception of meaning (see the beginning of the previous Section) harmonises with Hilbert's 'rational' conception of implicit definability, because accepted axioms of some branch of mathematics, together with the logical deduction-rules, govern the rigorous use of the primitive notions as they are actually used by the community of mathematicians in this branch. Hilbert's implicit definition can be taken to be a rigorous example of Wittgenstein's semantic grammar.

This is more-or-less the standard view among mathematicians of how primitive notions become meaningful: by axiomatising them, by defining them implicitly - although few mathematicians are aware, I believe, of how thoroughly Wittgensteinian the view of meaning is which is presupposed here.

So, we adopt axioms (and a formal language) of axiomatic set-theory in order to be as clear as possible about the meaning of the set-concept, because to adopt axioms is to adopt an implicit definition, and this is to fix the semantic grammar of the primitive vocabulary. Hallett seems to disagree:

Part of the problem is that 'set' (unlike 'aggregate' perhaps) is not an ancient, wellunderstood concept which can easily be taken as an axiomatic primitive in the knowledge that it can be supported by extra-axiomatic explanation. Unlike the case of natural number, this is largely why set-theory is axiomatised, because we do not understand the set-concept very well. (...) Where set-theory suffers as a foundation framework is that in general it does not bring this conceptual clarity with it.

(...)

The set-concept was always unclear, at least after the 'Cantorian transition'; and it was axiomatised in large part because of that unclarity (the paradoxes made a bad situation worse). ${ }^{11}$

We must arguably count Hallett among (a) the perplexity-mongers rather than among (b) the perplexity-exorcists. (a) The perplexity-mongers are prepared to let their perplexity dissolve iff the set-concept is reduced to "ancient, well-understood concepts", i.e. familiar words used standardly. For whatever reason, these mongers cannot bring themselves to accept a genuinely novel concept with novel rules governing its use; for them "conceptual clarity" apparently can never be accomplished in this manner. One wonders whether such a requirement for "conceptual clarity", i.e. to be reducible to "ancient, well-understood concepts", is compatible with the history of mathematics and with the history of thought generally. In mathematics, concepts such as a function, a group structure, a transfinite cardinal number, a large category and a functor were innovating and were eventually used

\footnotetext{
${ }^{11}$ Hallett [1984: 300, 305], sentence italicised by us. Cf. Section 2.4 .
} 
by mathematicians without them having the feeling they were stumbling around in some impenetrable conceptual fog, yet they had no reductions of the mentioned mathematical concepts to "ancient well-understood concepts". Conceptual innovations frequently consist in the acceptance of novel concepts that are not reducible to "ancient, well-understood concepts"; or if they are, then those reductions came historically after the reduced concepts already had proved their mettle. (b) In contrast to perplexity-mongers, perplexity-exorcists want to overcome the initial perplexity by understanding what has gone wrong. They compare the perplexity that arises in the mind when being confronted with an expression used nonstandardly to the physical shivering of your body when you plunge into the cold sea from a sunny beach.

We count Hallett among (a) the perplexity-mongers, because he has it backwards: he says that we need to understand the meaning of the set-concept (to attain "conceptual clarity") prior to the adoption of axioms in order to explain why we adopt them. I say we need not, because it is the other way around. Imagine we are at the end of the XIXth-century. Although we have some initial grasp of the novel Cantorian set-concept, we can openly assert we do not yet fully understand the novel set-concept (no "conceptual clarity" yet), but that we have definite intentions to go beyond what is reducible to familiar expressions used standardly and that we shall realise our intentions by adopting a novel semantic grammar. The meaning of the assertion 'we do not yet fully understand the meaning of the set-concept' we readily illustrate by admitting we feel insecure about the sethood of the whole of all ordinals (Burali-Forti antinomy), about the existence of the power-set of every set, or of the singletonset, or of the empty set; we feel insecure about which sets generally are supposed to exist, about the principle of full comprehension assigning a set to every predicate (Russell's antinomy), about what it means 'to combine ddots into a whole, into a single object' and to see 'a many as one object'. These feelings of insecurity spill over to various proofs which have been produced in set-theoretical practice so far. To remove these feelings of insecurity and perplexity, we shall have to make decisions about the mentioned issues.

No other road to conceptual clarity about the set-concept seems open, in particular when we accept it is a genuinely novel concept. We shall have to lay down its semantic grammar. Although Cantor's description cannot pass for an explication of the set-concept, we shall take it to lay down a rule of the semantic grammar of the set-concept. For the meaning of the set-concept is not some treasure we have to dig for in unknown soil but emerges from our use of the axioms (and the ensuing theorems) of set-theory, posterior to their adoption. Apparently Hallett finds the emerging meaning too shallow, because he still adjudicates the axiomatised set-concept to fall short of "conceptual clarity".

Further, I challenge the correctness of Hallett's claim that the concept of a natural number, a real number, a point in space, and what have you, are so much clearer and better understood than the set-concept. Frege, for one, thought it a scandal that no one knew what such an "ancient and well-understood" concept as a natural number was - he reduced them to extensions of predicates (Frege: "concepts") and regarded this reduction as an explication. Gödel, for another, literally subscribed to the converse; Wang [1996: 254] has reported 
Gödel saying: "Numbers appear less concrete than sets." No doubt Hallett will disagree and hold that the reductions of natural numbers to classes or to sets (the finite Von Neumannordinals and cardinals) are translations salva veritate all right, but certainly not explications. Now, even when we grant Hallett the supposition that the concept of a natural number is well-understood (pace Frege), then surely, we would like to conjoin, because we have played so many times language-games in which the concept of a natural number occurs. As a matter of fact from early childhood. We possess the capability to use it successfully and we do use it often; therefore we understand it so well. For numerous centuries mathematicians have played language-games wherein natural numbers occur and have proved all kinds of theorems about them. They are the most sophisticated players of this game. We know reasonably well what numbers are because of our endemic and unambiguous use of numbers, in spite of the fact that we may ask questions about them which do not readily have an answer.

What odd mind willingly accepts that a pair of socks and a couple of pheasants are instances - or examples or applications, whatever - of the number 2, but firmly rejects that a flock of sheep and a pack of wolves are instances - or examples or applications, whatever - of sets because 'many as one' and 'to combine into a whole' are unclear Expressions. As the variety of examples illustrates, in some respects the set-concept is just as ancient and well-understood as the natural number-concept. Has perplexity ever been evoked by shepherds when they talked about their flock of sheep? Have Indians ever been bedazzled by conceptual obscurity when they referred to a herd of bisons? Besides the similarities in use between the set-concept and flocks, herds, packs and what not, there are marked differences too, which make the set-concept comparatively novel from a historical perspective - this concept was of course not well-understood when Cantor began to use it. This situation is in principle not one scintilla different from that of the natural numbers: when we play, and we play successfully so that we come to know better and better how to play the game of sets, we come to understand the set-concept better and better, and then we shall know the meaning of the set-concept. This is what has actually happened in mathematics in the course of about one century.

The ardent players of the game of sets, i.e. professional set-theoreticians, model-theoreticians and mathematicians who study the continuum ('descriptive set-theory') and those who study transfinite arithmetic ('the higher infinite'), are usually more perplexed by the fact that others are perplexed by the set-concept rather than by the set-concept itself. This fact we submit as evidence in favour of the Wittgensteinian view defended here. The ardent users do not feel their minds clouded with "dense thickets of mystery"; not because they are 'insensitive to conceptual issues' or 'have no interest in philosophy', but because they are experienced users of the set-concept and communicate successfully. This means they know what it means. Any inclination to make illicit moves has petered out and concomitantly perplexity is no longer evoked. For them, the Expressions 'to combine into a whole' and 'a many seen as one' are considered, strictly speaking, redundant, or do not evoke perplexity at all. Of course, they have many unanswered questions about sets, their properties and relations, but these questions need not all be construed as indications of the alleged fact that 
they do not know what the set-concept means, anymore as the many questions that geologists have about mountains, their properties and relations need be construed as indications that geologists do not know what the word 'mountain' means.

Wang [1996: 254] has reported Gödel saying (author's emphasis):

Sets are multitudes which are also unities. A multitude is the opposite of a unity. How can anything be both a multitude and a unity? Yet a set is just that. It is a seemingly contradictory fact that sets exists. It is surprising that the fact that multitudes are also unities leads to no contradictions: this is the main fact of mathematics. Thinking a plurality together seems like a triviality: and this appears to explain why we have no contradiction. But 'many things for one' is far from trivial.

The multitarian whole of the set-concept is "far from trivial" because "seemingly contradictory", as Gödel asserts, but it appears not contradictory after all; the fact that we somehow can apprehend the set-concept, use it successfully and do not find it unclear, provides us with an explication of the absence of contradictions - "the main fact of mathematics".

To summarise, as soon as you are using words and expressions correctly, you are communicating successfully to your fellow set-theoreticians, as you are proving theorems in set-theory, as you are illustrating theorems by finding examples, as you are refuting conjectures by constructing counter-examples, as you are explaining theorems to others, and so forth, you understand the set-concept, you have knowledge of its meaning. This is how meaning emerges in language generally and the practice of set-theory is no exception.

In the current and the previous Section we have travelled a few miles on the road that will lead to the dissolution of multitarian wholism in Section 9. The next stop on this road consists in taking a more incisive look at the notion of implicit definability.

\section{Implicit Definability}

Let $\operatorname{Th}[\tau, P]$ be an axiomatised theory in a 1st-order language wherein term $\tau$ and predicate $P$ form the primitive vocabulary; and let $\operatorname{AxTh}[\tau, P]$ be the set of its axioms. ${ }^{12}$ As we have seen in the beginning of Section 4, Hilbert, then, proposed to take $\operatorname{AxTh}[\tau, P]$ as a implicit definition of the term $\tau$ and the predicate $P$. Nevertheless Hilbert, in the context of discussing his axiomatisations of geometry, wrote to Frege the following:

But it is surely obvious that every theory is only a scaffolding or schema of concepts together with their necessary connexions to one another, and that the basic elements [primitive vocabulary, FAM] can be thought of any way one pleases. If in speaking of my points I think of some system of things, e.g. the system: love, law, chimney-sweep ... and then assume all my axioms as connexions between these things, then my propositions,

\footnotetext{
${ }^{12}$ Thus by implication $\operatorname{AxTh}[\tau, P]$ is never empty.
} 
e.g. Pythagoras' theorem, are also valid for these things. In other words: any theory can always be applied to infinitely many systems of basic elements. ${ }^{13}$

At best this is a conjecture, and a rather speculative one for that matter. Suppose we take the basic elements to be human beings and the theory axiomatises a binary relation. One axiom says the relation is reflexive. Then it cannot be interpreted as 'is married to'. Suppose another axioms says it is transitive. Then it cannot be interpreted as 'is a parent of'. Axiom systems can clash with the semantic grammar of things and relations occurring in our natural language and this rules out that the axiomatised terms and predicates can be replaced with these terms and relations; if one does so, the axioms become false. If Hilbert's claim is that no matter what axioms are imposed on some primitive dyadic predicate think of $\in$ and ZFC, say - , there always is a binary relation to be found among human beings that makes the axioms true, then this claim is a conjecture at best. What human relation can stand for $\in$ so that the axioms of ZFC are truths about human beings? Let us gloss over Hilbert's speculation and suppose it is possible to interpret the primitive notions of some axiomatised theory in wildly differing ways - which presumably is all that Hilbert needs to make his point. For must we, then, not conclude that the implicit definition that the axiomatised theory provides is ambiguous? Does it not fail to single out a unique meaning that is assigned to every primitive notion? The answer seems in the affirmative, but for Hilbert, this ambiguity does not matter. Does this attitude, then, show that a particular view on the nature of mathematics is presupposed?

If one's view on the nature of mathematics is of the Formalist kind, then Hilbert's attitude is understandable. If one's view is of a Structuralist kind, then Hilbert's attitude is also understandable. I would like to suggest that no matter what view on the nature of mathematics one subscribes to, the kind of ambiguity that Hilbert tolerates, must be tolerated by meta-mathematical necessity. The reason is that even when we have a categorical axiomatisation of some branch of mathematics (such as 2nd-order set-theory and 2nd-order Peano arithmetic), so that all models of the theory are isomorphic and therefore semantically equivalent, the mentioned ambiguity remains. But a categorical axiomatisation is the very best one can have from a meta-mathematical perspective. If that is not good enough, then nothing ever will be. Ought implies can: it is unreasonable to criticise someone for having failed to accomplish the impossible.

When we draw a distinction between meaning and reference, and construe 'referent' modeltheoretically, we can say that an implicit definition fixes the meaning of a term, not its referents. ${ }^{14}$ Compare this to the meaning of the word 'mammal', which is clear, although its reference class contains wildly differing species. But even when we adopt the distinction betweem meaning and truth, it still does not follow that Hilbert's proposal stands unabashed. On the contrary, Hilbert's proposal to take $\operatorname{AxTh}[\tau, P]$ as the implicit definition of $\tau$ and $P$ as it stands, is unacceptable - although it does point in the right direction. In the remainder

\footnotetext{
${ }^{13}$ In Frege [1980: 40].

${ }^{14}$ The subject of Skolem trouble now comes in sight; we address it in the sequal paper, Muller [2004].
} 
of this Section, we discuss four Problems that any account of an implicit definability must solve and we shall observe in passing the Hilbert's proposal does not solve them all.

(A) No mathematical theory is born in an axiomatised form (between the birth of settheory, in 1879, to the first axiomatisation, in 1908, lies about 30 years). The axiomatisation of some branch of mathematics always occurs after a certain mathematical practice of proving theorems, formulating conjectures, constructing examples, \&c has established itself. This means that not just any implicit definition of the primitive notions of this practice will do. The implicit definition must, so to speak, 'save the linguistic phenomena of the mathematical practice under consideration', or more briefly, 'save the mathematical phenomena'. That is to say, the overwhelming majority of the established results must be vindicated rigourously by the axiomatisation of the mathematical practice that will serve as an implicit definition of its primitive concepts. If not, it will be rejected by the mathematical community as inadequate. We put it like this: the implicit definition must solve the Adequacy Problem.

(B) If we adopt an implicit definition of a certain notion, $\tau$ say, so as to fix the meaning of $\tau$, then $\tau$ must acquire the meaning that makes the implicit definition true, even analytically true, in the sense of true by virtue of meaning.

This seems plausible. But if the axioms of a theory are false, they fail to form an implicit definition of the primitive notions of the theory. For example, we cannot say that the false axioms of (an axiomatised version of) the chemical theory of phlogiston, or those of (an axiomatised version of) the physical theory of the luminiferous æther, implicitly define the terms 'phlogiston' and 'æther', respectively. But this is an odd consequence, because we are interested in the meaning of the primitive notions, not whether the ensuing theories are true or false, or whether their primitive terms refer or not. So what to do with false theories? This is what we call the Truth Problem.

(C) What guarantee do we have that there is a meaning which the implicitly defined notion will acquire? We call this the Existence Problem. ${ }^{15}$

The Existence Problem was raised rather urgently by A.N. Prior [1960]. Suppose we consider the introduction- and elimination-rule for conjunction in Gentzen's system of natural deduction as constituting an implicit definition of the word 'and'. Now imagine we implicitly define a new logical connective, called tonk and denoted by 2 , by means of the following introduction- and elimination rule:

$$
\psi \vdash \psi \prec \varphi \quad \text { and } \quad \psi \imath \varphi \vdash \varphi,
$$

where $\varphi$ and $\psi$ are arbitrary sentences of the language under consideration.

When we accept tonk, we can, by successive application of the rules (1), prove that anything entails anything: $\psi \vdash \varphi$. Therefore Prior called tonk 'the runabout inference-ticket'. Since $\psi$ and $\varphi$ are arbitrary sentences, we can substitute a theorem of logic for $\psi$ and a

\footnotetext{
${ }^{15}$ One can strengthen this to a 'Unique Existence Problem', asking for a guarantee there exists precisely one meaning. It is not difficult to argue there is no need to require there be precisely one meaning. Depending on the context, ambiguities can be tolerated without any detriment to the use in that context.
} 
contradiction for $\varphi$; thus the introduction of tonk in the language renders every theory formulated in that language inconsistent. Remember we have to save the linguistic phenomena (A). In the context of set-theory, the prominent phenomenon is that the participants are engaged in proof and disproof. To makes sense of that an interesting deductive apparatus is needed, say 1st-order predicate logic. Due to ex absurdum sequitur quodlibet, even a single contradiction is then probited to make its appearance, otherwise we cannot possibly save the linguistic phenomena of proof and disproof (A). Hence inconsistencies are totally unacceptable. The conclusion is, then, that there is no meaning available for the alleged new logical connective tonk (1). Hence not every group of sentences qualifies as an implicit definition that successfully assigns meaning to its primitive vocabulary. How to separate the ones that do from the ones that do not? Is requiring consistency enough? Answering this question we take, in Wittgensteinian vein, to solve the Existence Problem.

(D) If we have solved the Existence Problem (C), we should also be able to explain how the term comes to possess the meaning that is singled out by the implicit definition. We call this the Explanation Problem. Horwich [1998: 135] says:

You cannot make a wall red by saying 'Let it be red!' — you have to paint it.

To address the Explanation Problem, it becomes unavoidable to say something about how meaning emerges generally and to connect this to the notion of implicit definability. We do this in the next Section, after we have given an account of implicit definability that solves (A) the Adequacy Problem, (B) the Truth Problem, (C) the Existence Problem and (D) the Explanation Problem; and then to argue that the axioms of ZFC, say, fit this account so that we can justifiably assert they define the set-concept implicitly.

\section{Semantic Deflationism}

Although Wittgenstein avoided building a philosophical theory of meaning like the plague, very few will doubt that Wittgenstein had some coherent conception of meaning in mind, on the basis of which he performed his analyses and from which he launched his criticisms. In his book Meaning [1998], P. Horwich expounds a theory of meaning which he has baptised semantic deflationism; it is intended to clarify Wittgenstein's conception of meaning (and truth) and to support it. ${ }^{16}$

Semantic deflationism, as we understand it, counts five postulates (warning: some terminology and simplifications are ours).

\footnotetext{
${ }^{16}$ Horwich's claim that his theory of meaning is Wittgensteinian in that it is a use-theory seems incontestable in the light of the quotation of Wittgenstein at the beginning of Section 3 (such quotations can be provided ad libitum whereas no quotations of Wittgenstein II can be provided to the contrary). Whether Horwich's use-theory is the best explanation of Wittgenstein's writings on meaning is an exegetical claim (not explicitly defended by Horwich) which is irrelevant for this paper (and for Horwich's theory of meaning too, for that matter).
} 
I. Concept Postulate. Meanings are concepts and concepts are abstract objects.

II. Use Postulate. For every expression there is a 'small' submanual of its use manual that constitutes its meaning; this submanual we call the 'meaning-manual' of the expression.

III. Synonymy Postulate. Synonyms have the same meaning-manuals.

IV. Truth Postulate (Convention T). For any proposition $\mathrm{P}$, ' $\mathrm{P}$ ' is true iff $\mathrm{P}$.

V. Reference Postulate. The propositional content of term $\tau_{b}$ (usually: ' $b$ ') designates object $c$ iff $b$ is identical to $c$. Term $\tau_{b}$ refers to object $c$ iff there is some term, $\sigma_{a}$ say, such that: $\sigma_{a}$ and $\sigma_{b}$ have the same propositional content, and $\sigma_{a}$ designates $c$.

Since postulates III and V will play no part in this paper, we can afford to ignore them (we merely included them here for the sake of completeness). Next follow a few explanatory comments on postulates I and II in so far as is necessary to understand the application we shall make of.

Addendum I. The assertion that meanings are abstract objects is merely to distinguish them from concrete objects - no transcendent realm of meanings is or needs to be posited. The meaning of a linguistic expression, $\tau$ say, is a property of that expression, denoted by $M(\tau)$; moreover this property is a concept, which in turn is an abstract object. This stands in marked contrast to other theories of meaning, where meaning is a binary relation: ' $\tau$ means $t^{\prime}$, or 'the meaning of $\tau$ is $t^{\prime}$, is then construed as there being a relation, $R$ say, that holds between $\tau$ and $t$, by virtue of which $t$ is the meaning of $\tau$ :

$\tau$ means $\mathrm{t}: R(\tau, \mathrm{t})$.

Unlike (2), Horwich construes this as follows:

$\tau$ means $\mathrm{t}: M(\tau)=\mathrm{t}$,

where $t$ is a concept. Horwich [1998, passim] says things like: 'cat' means CAT, i.e. $M($ cat $)=$ CAT.

Addendum II. In order to be able to understand the Use Postulate, we need to understand (i) what a 'use manual' is, and (ii) what 'to constitute' means.

(i) The use of some expression $\tau$, say, is the sum-total of all contexts in which some expression is spoken or written that contains $\tau$ (over some period of historical time). We can label the contexts, denoted by $\mathcal{C}_{j}(j \in \mathbb{N})$, and the concomitant expressions so as to make ordered pairs of type

$$
\left\langle\mathcal{C}_{j}, L_{j}[\tau]\right\rangle
$$


where $L_{j}[\tau]$ is a list of expressions $\sigma_{0}[\tau], \ldots, \sigma_{k}[\tau]$ that contain $\tau(k \in \mathbb{N})$. So the idea is the following $(l=0,1,2, \ldots, k)$ :

In context $\mathcal{C}_{j}$, the usage of any expression $\sigma_{l}[\tau] \in L_{j}[\tau]$ is appropriate .

(The symbol $\mathcal{C}_{j}$ can be a name of particular context or a variable ranging over a certain type of context.) We collect all the ordered pairs (4) in:

$U(\tau)$ : the use manual of expression $\tau$.

List $L_{j}[\tau]$ will generally contain many expressions, because quite a number of expressions containing $\tau$ will be appropriate in the given context and therefore all belong to the usemanual. Similarly, the same expression can be used on different occasions, e.g. 'This is good', 'That is big', so that it will occur in many lists of different contexts. The meaning of a word like 'and' will be the same in all contexts; 'and' can therefore be said to have a transcontextual or absolute meaning. In contrast, the meaning of the word 'big' will depend heavily on the context (it means something different in nano-technology than in cosmology); so 'big' has a contextual meaning.

A substantial number of contexts consists in someone else using language. For this is how we talk to each other. This is how communication arises. Call a submanual of $U(\tau)$ a set of contexts occurring in $U(\tau)$, each one paired with a sublist from the list occurring in $U(\tau)$.

(ii) The notion of 'to constitute' is supposed to be the familiar one, as in: 'consisting of $\mathrm{H}_{2} \mathrm{O}$-molecules' constitutes 'being water', and 'emitting or reflecting light of wavelengths about 600 nanometer' constitutes 'being yellow'. Hence 'to constitute' seems a relation between properties, which we describe by predicates. Let $F$ and $G$ be two predicates, which need not have the same logical form. Horwich defines [1998: 25]:

$$
\begin{array}{r}
F \text { constitutes } G \text { iff } \quad \text { (a) } F \text { and } G \text { apply to the same things; and } \\
\text { (b) } F \text { and (a) explain all facts about } G .
\end{array}
$$

The predicates 'being water' $(G)$ and 'consisting of $\mathrm{H}_{2} \mathrm{O}$-molecules' $(F)$ apply to concrete objects (a); and facts about $\mathrm{H}_{2} \mathrm{O}$-molecules and about what happens when you take lots of them together explain the facts of water, e.g. its liquid state at room temperature, transparency, viscosity, adhesion and the capability to solve many chemical substances (b).

Now we can understand what is being asserted in the Use Postulate: every expression has a 'small' submanual that constitutes its meaning. This meaning manual is supposed to explain the overall use of the expression; it should determine the use manual; it should be taken, we add, as Wittgenstein's semantic grammar. But how small is 'small'? There is no point in fixing a number; but the smaller, the more bite the theory of meaning under consideration will have. Horwich [1998: 45] provides examples of words whose meaning manual counts a few items only, such as the logical connectives and the quantifiers; for these, he simply takes the introduction- and elimination-rules from Gentzen's System of Natural Deduction to be their meaning manuals. 


\section{Implicit Definability Revisited}

The account of implicit definability we are about to offer is a slight variation on Horwich's account [1998: 138]. Like Horwich's account, our account is given in the framework of semantic deflationism; but it is logically stronger (see below).

Suppose we have some mathematical practice that has recently established itself as a legitimate branch of the tree of mathematical knowledge. Suppose further, for the sake of simplicity, that the practice contains one novel term, $\tau$, and one novel predicate, $P$ - all the other notions used are familiar and unproblematic. Suppose an axiomatisation $\operatorname{AxTh}[\tau, P]$ is proposed and accepted. From the mathematical practice we draw up a list of sentences in which $\tau$ and $P$ occur, called $L[\tau, P]$, that we intuitively consider to be constitutive for the meaning of $\tau$ and $P$. This is our set of paradigm linguistic phenomena, or in this case more appropriately, mathematical phenomena, that $\operatorname{Th}[\tau, P]$ must save, i.e. $L[\tau, P]$ must be a set of theorems deducible from $\operatorname{AxTh}[\tau, P]$.

Now we are able to formulate our

Implicit Definability Criterion. A set of axioms implicitly defines the meaning of its primitive vocabulary iff (Cr1) it is logically possible that the axioms are true and $(\mathrm{Cr} 2)$ they save the paradigm linguistic phenomena.

A few systematic comments on this Criterion are in order.

First, when we take Hilbert to assert that axioms are implicit definitions that provide primitive vocabularies with meaning iff they are consistent, and when we further take Hilbert's view into account that for mathematical theories consistency implies truth, his notion implies the following version, which became the standard account of implicit definability until Horwich proposed his account: a set of axioms standardly implicitly defines the meaning of its primitive vocabulary iff they are true. ${ }^{17}$ Horwich's weakening of this standard account consists in weakening 'true' to 'possibly true'. ${ }^{18}$ When we construe truth model-theoretically, then possible truth is the same as having a model, and when we further take 1st-order predicate logic as our background logic, then Horwich's notion coincides with Hilbert's original notion.

For us, (Cr1) logically possible truth (consistency, say) is necessary, like it is for Hilbert and Horwich, but not sufficient, unlike Hilbert and Horwich; we require in addition (Cr2)

\footnotetext{
${ }^{17}$ For lovers of paraconsistency, an obvious revision of the implicit-definability Criterion is readily available. Hilbert in a letter to Frege (in Frege [1980: 42], our emphasis): "If the arbitrarily chosen axioms together with all their consequences do not contradict one another, then they are true, and the things defined by the axioms exist." On another occasion Hilbert said that consistency is not enough for a theory to be acceptable by the mathematical community; the presence of success of a theory must be added; cf. Hilbert [1925: 163]. One can still maintain, however, that even unacceptable mathematical theories can be meaningful, even those having some success, such as finite Set-Theory, which is unacceptably restrictive but surely perfectly meaningful.

${ }^{18}$ Horwich [1998: 138] says "can be regarded as true". Does he have a stronger - or perhaps a weaker modality than 'logical' in mind?
} 
that the implicit definition has to save the paradigm linguistic phenomena. If condition ( $\mathrm{Cr} 2)$ was a tacit requirement of Hilbert (cf. endnote 17), and also of Horwich, then our Criterion only has the additional virtue of making this tacit requirement explicit.

Secondly, our Criterion, just as the other criteria formulated above, implies that inconsistent theories never implicitly define their primitive vocabulary.

Thirdly, for the sake of clarity we emphasise that our Criterion can only be applied when an axiomatisation and a list of paradigm mathematical phenomena are available.

Next we argue that our Criterion solves the four Problems (A)-(D) raised in the previous Section. This is our ground for accepting it.

(A) The Adequacy Problem is that not just any list of axioms qualifies as an implicit definition; the last-mentioned must 'save the mathematical phenomena' which occur in the practice. Any list of axioms that qualifies as an implicit definition of its primitive vocabulary has solved this problem owing to our second conjunct in our Criterion. Admittedly our Criterion solves the Adequacy Problem 'by construction', but none of the other available criteria for what it takes to be an implicit definition solves it at all. ${ }^{19}$

(B) The Truth Problem was solved by Horwich by weakening 'truth' to 'possible truth', or consistency for that matter. For this permits us to say that also actually false yet possibly true theories, such as the chemical theory of phlogiston and the physical theory of the luminiferous æther, implicitly define their primitive vocabulary.

There is another solution to the Truth Problem. All the examples of false theories of which we also want to say that they define their primitive vocabulary implicitly, are scientific and not mathematical (the theory of phlogiston, the theory of luminiferous æther, Kelvin's theory of knot-molecules). All these terms (are supposed to) refer to concrete objects. So for the meaning of a word denoting an abstract object, e.g. 'set', we could still require that the implicit definition is an analytic truth about sets. One big difference between science and mathematics is that by means of experience we have some direct epistemic access to the world of concrete objects which allows us to test scientific theories, whereas it is controversial whether there exists a world of abstract objects, let alone whether we have some direct epistemic access to this world which allows us 'to test' mathematical theories in a sense even remotely similar to the case in science. This means that when we are concerned with the implicit definition of an abstract concept such as the set-concept, we can still require that $\left(\mathrm{Cr}^{\prime}\right)$ the axioms of set-theory are analytic truths of sets, rather than requiring $(\mathrm{Cr} 1)$ they are possibly true. Then the axioms of different set-theories are analytic truths of different set-concepts; such set-concepts are the same in so far as their theories share axioms and theorems. Replacing $(\mathrm{Cr} 1)$ with $\left(\mathrm{Cr}^{\prime}\right)$ for abstract concepts in the Implicit Definability Criterion is obviously compatible with Nominalism; it is also compatible with a pluralistic Platonism, which posits the existence of different universes of sets, each 'described' by the different axiomatic set-theories. It is not compatible with a variety of Platonism that posits

\footnotetext{
${ }^{19}$ If one takes the view that the given axiomatisation has already solved the Adequacy Problem, then one has to expound an account of what an axiomatisation is such that this is guaranteed. That is possible, but it takes more journal space. In the end it will boil down to the same thing as we are proposing.
} 
a single universe of sets, so that only one of the axiomatic set-theories we have can be true; such a Platonist can endorse the current Criterion.

(C, D) As in Horwich's criterion, the presence of the logical possibility of truth avoids the devastating consequences such as Prior's [1960] 'runabout inference-ticket'. ${ }^{20}$ If AxTh $\left.\tau, P\right]$ qualifies as an implicit definition, then it also qualifies as the meaning manual $U_{\mathrm{M}}(\tau, P)$ of term $\tau$ and predicate $P$, with as context the mathematical practice under consideration, because every rigorous usage can be explained on the basis of this meaning manual. The Use Postulate of semantic deflationism then guarantees the existence of meanings, which means that the Existence Problem is solved (C). But the Use Postulate also provides us with an explanation of how implicit definitions succeed in this: because as a meaning manual, from which the entire use manual follows, the implicit definition constitutes (7) and thereby explains the meaning (this is what the Use Postulate asserts). Whatever we consider as facts about the meaning of the set-concepts, they will have to explained by the meaning-manual, otherwise the Use Postulate is not fulfilled. The most prominent facts are precisely those listed in $L[$ set, $\in]$; they are addressed at length in Section 9.

Now that we have a satisfactory Criterion of implicit definability, our task will be completed when we have established that the axioms of Zermelo-Fraenkel set-theory (ZFC), say, meet this Criterion. Before we do this (in Section 9), let us end this Section by devoting a few sentences to how the Criterion relates to philosophical views on mathematics.

Views on mathematics (philosophy of mathematics) and views on meaning (philosophy of language) generally seem orthogonal to each other: any view on mathematics (Logicism, Formalism, Intuitionism, Structuralism, Platonism, Nominalism) seems compatible to any view on meaning, although some particular view of meaning seems to go naturally with some particular view of mathematics. For example, a reference-conception of meaning seems to go well with both Intuitionism and with Platonism, because we can take the mathematical concepts to refer to mental processes and abstract entities, respectively. As another example, a use-conception of meaning such as semantic deflationism, and specifically the concept of implicit definability developed here within the framework of semantic deflationism, seems to go well with both Formalism and Nominalism. I think that Formalism appears a less sterile view of mathematics when looked upon from the perspective of semantic deflationism - or any other use-conception of meaning for that matter. The reason is that 'playing games with meaningless symbols' is not a sterile activity at all. As soon as we realise that symbols in mathematics (often) stand for concepts, and that providing axiomatisations is tantamount to providing implicit definitions, we see how mathematics boasts of meaning. The clarity and often exhaustiveness of the axiomatisations provided, which quickly leads to consensus in the community of (the relevant) mathematicians, explains the

\footnotetext{
${ }^{20}$ For a more detailed explanation, see Horwich [1998: 137-150]. Horwich's move from actual truth to possible truth also solves a few other, minor problems raised by the standard account. We gloss over them here, but would like to mention that by implication our strengthening of Horwich's notion solves these other problems too.
} 
clarity of the meaning of mathematical concepts. We leave this side issue now.

\section{Attack of the Neo-Fregeans}

B. Hale and C. Wright, two of the spear-heads of a Neo-Fregean programme in the philosophy of mathematics, have criticised Horwich's conception of implicit definition severely. We explain their "decisive objection" and argue it is incoherent in a sense that will become evident as we proceed (in Hale \& Wright [2001: 119-126]).

Hale and Wright begin by submitting that Horwich "succumbs to dangerous associations" evoked by semantic deflationism, namely that singling out a meaning for a given expression is

... slipping into a picture - which will strike anyone but an extreme realist about meanings as tendentious - of implicit definition as aimed at hitting on some (unique) preexisting meaning, as if it were akin to an attempt at reference-fixing. ${ }^{21}$

The first thing to remark is that a philosopher engaged in semantic deflationism is not committed to believe in some pre-existing realm of meanings, anymore than a mathematician engaged in set-theory is committed to some pre-existing Platonic realm of abstract entities called 'sets' - which will strike anyone but an extreme realist about abstract entities as tendentious. The analogy between meaning-assignment and reference-fixing can be drawn, but it need not be drawn. Just as one can resist a realistic interpretation of the inhabitants of the domain of discourse of set-theory, one can resist a realistic interpretation of the inhabitants of the domain of discourse of semantic deflationism. In semantic deflationism, to call meanings 'abstract objects' merely serves to delineate them from 'concrete objects'. Therefore any criticism of semantic deflationism that somehow relies on the analogy between meaning-assignment and reference-fixing cannot be convincing. We are not sure how strongly the criticism of Hale and Wright relies on this analogy, but their running analogy to finding the meaning of an expression is to find the referent of 'Jack the Ripper' and it seems that semantic deflationism is not committed at all to this misleading analogy.

The heart of their criticism reads that Horwich cannot solve what they call 'the understanding problem': Horwich "merely fixes a meaning without explaining it" ([2001: 124]). Let me quote this criticism (while leave out their distracting, discursive interjections):

It is a critical problem [the understanding problem, FAM] — on the conception of implicit definition on which Horwich is working. For think what would be required to resolve it in a particular case. In order to identify the referent of 'Jack the Ripper' (...) an agent will have to determine who committed the crimes in question, that is, will have bring the perpetrator under some canonically identifying concept: 'the 5th from the left in

\footnotetext{
${ }^{21}$ Hale \& Wright [2001: 121], their italics.
} 
that line-up', for instance, or 'the Prince of Wales'. By analogy, in order to arrive at an understanding of the meaning of ' $\tau$ ' (...) a thinker will have to bring the referent of 'that (unique) meaning which suffices for $\tau$-ness' to express a truth under some canonically identifying concept: that is, identify it as the referent of some expression whose own meaning (...) serves to disclose what meaning it is. But that is tantamount to the demand that successful implicit definition requires a recipient to have (...) independent resources sufficient for an explicit definition of the definiendum. Yet it was all along an absolutely crucial point about implicit definitions (...) that they were to serve in cases (...) where no resources for (...) explicit definition were available. Horwich's model is thus in tension with this absolutely crucial point. We take that to be a decisive objection to it. ${ }^{22}$

Generally, if some worry is raised about a certain view that cannot be answered without denying a presupposition of the problem the view is supposed to solve, one need not reject that view, because there is always the possibility of denying that the view gives rise to that worry. In fact, if denying a presupposition is the only answer to the worry, one can argue that the worry is a mistake. In the present case, if providing an implicit definition of expression $\tau$ raises a worry which can only be solved by providing an explicit definition of $\tau$, this may be a reason to reject that worry as a mistake. We believe that Hale and Wright have made such a mistake. We explain.

Succinctly, Hale and Wright's reasoning seems to proceed as follows: even if we have the guarantee that $L[P, \tau]$ fixes meanings $\mathrm{P}$ and $\mathrm{t}$, we must know what $\mathrm{P}$ and $\mathrm{t}$ are. In order to know that, we need to have unproblematic expressions in our language, say $Q$ and $\sigma$, such that $M(Q)=\mathrm{P}$ and $M(\sigma)=\mathrm{t}$, because trivially we only have access to meanings by means of language. But now, due to $M(Q)=M(P)$ and $M(\sigma)=M(\tau), Q$ and $\sigma$ define $P$ and $\tau$ explicitly!

The mistake in this reasoning is the step from 'we must know what $\mathrm{P}$ and $\mathrm{t}$ are' to 'we need to have unproblematic expressions $Q$ and $\sigma$ in our language'. The supposed fact that $L[P, \tau]$ is an implicit definition of $P$ and $\tau$, exactly implies that we cannot come to know the meanings of $P$ and $\tau$ by means of synonymous expressions available in our language, $Q$ and $\sigma$ say. If we were able to find such expressions $Q$ and $\sigma$, there would be no need to come up with an implicit definition because we then would have explicit definitions. The right conclusion would then have been that we were mistaken in assuming that $P$ and $\tau$ have to be defined implicitly. But per assumptio we consider cases where no such mistake is made, situations where no such $Q$ and $\sigma$ actually are available. Hence Hale and Wright essentially charge Horwich for being unable to solve an impossible problem: it is impossible to provide an explicit definitions for expressions that do not have explicit definitions. The charge is incoherent. With Celsus we hold that impossibilium nulla obligatio est.

To reiterate, the meanings $P$ and $t$ are obtained by means of the Use Postulate of semantic deflationism via the relevant meaning-manual, which contains only sentences from our

\footnotetext{
${ }^{22}$ Hale \& Wright [2001: 125]. I have replaced their ' $f$ ' with our ' $\tau$ '.
} 
language in each of which $P$ or $\tau$ occurs, or both occur.

\section{The Fall of Multitarian Wholism}

In order to assert that the axioms of ZFC, denoted by AxZFC[set, $\in]$, qualify as an implicit definition of the set-concept and the membership-relation, we must, according to our Criterion of implicit definability, establish that ZFC (Cr1) is possibly true and $(\mathrm{Cr} 2)$ that it saves the paradigm mathematical phenomena of the practice of set-theory.

(Cr1) It certainly is not obvious that ZFC possibly is a true theory about sets, because it seems that the only way to convince ourselves of this is to produce a consistency proof, provided model-theoretic truth is sufficient for truth. Yet an informal argument for the consistency with this provision can be mounted. This informal argument starts by asserting that the axioms of ZFC are supposed to be true of sets, if anything. Whatever the meaning of the word 'set' is, the axioms of ZFC must be true of it. They are, in fact, analytic truths about the set-concept: the axioms of ZFC are true of the set-concept because they single out the meaning 'set' must have in order to make them true. ${ }^{23} \mathrm{Next}, \mathrm{ZFC}$ is closed under 1st-order deduction, which is demonstrably sound. So all theorems of ZFC are analytically true. We certainly know at least one sentence in the language of ZFC that is not a theorem of ZFC. We now conclude that ZFC is a consistent collection of analytic truths about the set-concept - a conclusion that is actually stronger than we need in order to meet (Cr1). Of course, by meta-mathematical necessity we cannot formalise this argument (Gödel). Yet as an informal argument it makes sense.

There is also a pragmatic argument for the consistency of ZF (from which the possible truth of ZFC follows), which runs as follows. ZF has been explored almost to the point of exhaustion, both mathematically as well as meta-mathematically, so that if it were to harbor some contradiction, it would have been found by now. (Remember that when Russell in 1903 discovered his famous contradiction in Frege's theory, this theory had, at the time, only been fully explored by its creator; so the second scrutinous explorer who came along already stumbled upon a contradiction. Compare this to the legions of mathematicians and logicians who have explored ZF over the past century.) The results of the practice of proof and disproof in set-theory supports in toto the conjecture that ZF is consistent.

We next concentrate our efforts on ( $\mathrm{Cr} 2)$, the composition of a list of paradigm mathematical phenomena of the practice of Cantorian set-theory - of course as the practice was around 1900.

(Cr2) Here comes the list we call $L[$ set, $\in]$.

1. Sets are objects.

\footnotetext{
${ }^{23}$ We remark that we would not like to assert this in all cases, because of the possible falsehood of the axioms; cf. Section 7, (B) the Truth Problem. But hic et nunc, with this particular concept and this particular theory, we would like to assert this.
} 
2. A set is the result of combining objects, e.g. other sets, 'into a whole'.

3. A set is 'a many' thought of 'as one object', save singleton-sets and the empty set $(\varnothing)$, which cannot be thought of this way.

4. A set is the kind of thing that has members, save $\varnothing$.

5. A set is constituted by its members: every assertion about a particular set must ultimately involve its members.

6. A set is constituted by its members: two sets are identical iff they share all their members.

7. Every set has subsets and every non-empty set has proper subsets.

8. With every two sets we can make the union-set, which generally is larger than either one; and we can make the intersection-set and complement-sets, which are generally smaller than either one of them.

9. Every set is either finite or transfinite.

10. Every transfinite set is either denumerable or non-denumerable.

11. Some proper subset of a transfinite set can be bijected to it.

12. The power-set of a set is a set and is larger than it; but its union-set need not be.

13. No set is self-membered $(X \notin X)$; there is no Siamese twin-set $(X \in Y$ and $Y \in X)$; there is no Narcissus-set ( $N=\{N\}$, this set is full of itself); and there are no infinitely descending membership-chains $\left(X_{1} \in X_{2} \in X_{3} \in \ldots\right)$.

14. Every set has a unique cardinal number.

15. There is such a thing as 'cardinal arithmetic', which coincides with the arithmetic as we know it if we restrict ourselves to finite cardinals.

16. Sets have the same cardinal number iff they are equinumerous (they can be bijected to one another); a set is cardinally smaller than another set iff it can be injected into it but not vice versa), and larger iff it can be surjected onto it but not vice versa.

17. Every set is countable in-the-generalised-sense of being well-orderable; every wellordered set has a unique ordinal number.

18. There is such a thing as 'ordinal arithmetic', which coincides with the arithmetic as we know it if we restrict ourselves to finite ordinals.

19. For every two non-equinumerous sets it holds that one is larger than the other, or vice versa. 
20. Every finite and every transfinite set is increasable, i.e. there is another set which includes it and is larger than it.

21. The cumulative hierarchy exhausts the domain of discourse of set-theory.

We have attempted to make an exhaustive list, but a few sentences more or less in this list will not affect the point we are trying to make (add your own favourite meaning-constitutive sentences if you like - in general, lists like these may change over historical time as the subject evolves). The point is that some list like $L[$ set, $\in]$ has to be drawn up in order to solve (A) the Adequacy Problem (see Section 5) and then show that AxZFC[set, $\in]$ constitutes the meaning of the set-concept, because $L[$ set, $\in]$ comprises the most prominent facts about the meaning of 'set' to which the Use Postulate refers via the definition of 'to constitute' (7). We have drawn up this list of paradigm mathematical phenomena by observing first and foremost the mathematical practice of Cantorian set-theory, because there (if anywhere) we find expert users. As soon as we adopt the axioms of ZFC, and we translate the sentences 1-21 in the list into $\mathcal{L}_{\epsilon}$ faithfully, we recover every single sentence in the list as a proved theorem:

$$
\mathrm{ZFC} \vdash L[\text { set, } \in]
$$

(not conversely, of course, but that was never required anyway). This should be evident, except for items 1, 2 and 3; they deserve a special treatment because of the worries of Section 2 and we shall turn to these items shortly - also 15 and 18 need a bit of fleshing out, but we gloss over it because it is unproblematic. At this point a remark on the continuum hypothesis is in order.

Should we add to our list that the continuum hypothesis must be proved or disproved? This 'decidability' might be considered as a requirement for the adequacy of set-theory (as both Cantor and Hilbert indeed required). From the meaning of the set-concept, however, should not necessarily flow such a sophisticated sentence about the interrelation of certain specific cardinal numbers, which is what the continuum hypothesis is. Like so many sophisticated sentences of $\mathcal{L}_{\in}$ that turn out to be undecidable, it should not be taken as constitutive for the meaning of the set-concept. We now turn to items 1, 2 and 3.

Statement 1 of our list says that sets are objects. It should be obvious that, indeed, ZFC treats sets as objects: there are set-variables and set-names in $\mathcal{L}_{\in}$ and they are the logical subjects which get predicated. In this sense Statement 1 is vindicated.

Statements 2 and 3 are the crucial Expressions 'a many as one object' and 'to combine into a whole', respectively, that gave rise to the thesis of Multitarian Wholism (end of Section 2). In Section 3, we have explained that the perplexity these Expressions evoke results from confusing different rules that govern them in different contexts. In the examples of 'as one', these contexts were: describing a painting and the experience of looking at it, and similarly for a piece of music and a work of literature. In the examples of 'to combine into a whole', these contexts were: modelling an astronomic phenomenon, carpentry, solving a problem in arithmetic, strategic deliberations of the military, and politics, respectively. We concluded 
Section 3 by saying that we must formulate the rules of the semantic grammar of the crucial Expressions such that Cantor can be said to have followed these rules. This is precisely what we shall do next. It leads to the fall of multitarian wholism.

So we are going to define the paradigm Expressions in 2 and 3 in $\mathcal{L}_{\epsilon}$. We say that $n \geqslant 2$ sets $X_{0}, X_{1}, \ldots, X_{n-1}$ are combined into whole $S$ iff they are all and the only members of set $S$; and that $n$ elements $X_{0}, X_{1}, \ldots, X_{n-1}$ are seen as one object iff there is a set that is a whole into which they are combined:

$$
\begin{aligned}
& \operatorname{CombWhole} 1\left(X_{0}, X_{1}, \ldots, X_{n-1}, S\right) \equiv \forall Y\left(Y \in S \longleftrightarrow \exists j \in I_{n}: Y=X_{j}\right), \\
& \text { SeenAsOne1 }\left(X_{0}, X_{1}, \ldots, X_{n-1}\right) \equiv \exists S: \operatorname{CombWhole1}\left(X_{0}, X_{1}, \ldots, X_{n-1}, S\right),
\end{aligned}
$$

where $I_{n} \equiv\{2,3, \ldots, n-1\}$. Since no rules are adopted for the cases $n=0$ and $n=1$, the definitions above do not apply to the empty set and to singleton-sets. Any player of axiomatic set-theory who now speaks of sets $\varnothing$ and $\{\mathbb{N}\}$, say, as 'combined into a whole' and 'a many as one', respectively, is from now on literally uttering nonsense. (One can also accept $\varnothing$ and singleton-sets as 'degenerate cases' by no longer requiring that $n>1$.) So the worries raised by Frege and Black (Section 2) dissolve: neither $\varnothing$ nor $\{X\}$ is 'a many seen as one' in the only sense now available in $\mathcal{L}_{\epsilon}$, which is defined in (9).

Evidently, rules (9) work only for a finite number of elements. But when these definitions are considered satisfactory, they do in principle apply to our troublesome example of the sixteen tomatoes (Section 2). To encompass also the infinite case, we adopt a rule with a meta-mathematical flavour: all sets for which predicate $\varphi(\cdot)$ in $\mathcal{L}_{\epsilon}$ holds are combined into whole $S$ iff $S$ is the existing set-extension of $\varphi(\cdot)$; and these sets are seen as one object iff there exists a whole into which they are combined:

$$
\begin{aligned}
& \text { CombWhole2 }(\varphi(\cdot), S) \equiv \forall X(\varphi(X) \longleftrightarrow X \in S) \wedge \# S \geqslant 2, \\
& \text { SeenAsOne2 }(\varphi(\cdot)) \equiv \exists S(\forall X(\varphi(X) \longleftrightarrow X \in S) \wedge \# S \geqslant 2) .
\end{aligned}
$$

The formal connexion between (9) and (10) consists in considering for $\boldsymbol{\varphi}(\cdot)$ in (10) the disjunction: $Y=X_{0} \vee Y=X_{1} \vee \ldots \vee Y=X_{n-1}$.

It serves to mention that the two definitions in (10) are equivalent in the following sense:

$$
\text { ZFC } \vdash \text { SeenAsOne2 }(\boldsymbol{\varphi}(\cdot)) \longleftrightarrow \exists S: \text { CombWhole2 }(\boldsymbol{\varphi}(\cdot), S) \text {. }
$$

Whether SeenAsOne2 $(\varphi(\cdot))$ and $\exists S:$ CombWhole2 $(\varphi(\cdot), S)$ are theorems of ZFC depends of course on $\varphi(\cdot)$ (and on $S$ in the second case); as general theorem-schemata they fail. When $S$ is, however, a subset of some existing set, $Z$ say, then there always is, by virtue of Zermelo's Axiom-Schema of Separation, a set-extension of: $\varphi(X) \wedge X \in Z$. Our charting of terra incognita has now come to an end.

Let us be perfectly clear about the status of definitions (9) and (10). They are neither meant to be explicatory in the sense that the informal troublesome Expressions are reduced to ancient, well-understood concepts, nor are they supposed to coincide with the informal descriptions we provided in Section 2. They are meant as new rules governing expressions 
in $\mathcal{L}_{\epsilon}$ that lie as close to the informal Expressions as we can possibly get in $\mathcal{L}_{\epsilon}$. When we thus take Cantor's description of a set as anticipating this, rather than as an explication of the set-concept, we can take his 'definition' to be fully vindicated.

The perplexities we experience when asked to ponder the meaning of the crucial Expressions are now considered as a result of a confusion of meanings. When we hold on firmly to $\mathcal{L}_{\epsilon}$ and to set-theory, enriched with definitions (9) and (10), and we refuse to tread beyond these sharply delineated bounds of meaning, then perplexity will not be evoked. But as soon as we make an illicit move, by confusing definitions ('rules') (9) and (10) with the not-so-rigourously delineated semantic rules that govern the expressions 'many as one' and 'to combine into a whole' in a variety of other contexts (notably the examples we presented above), then we are producing nonsense by mixing meanings.

We conclude that AxZFC[set, $\in$ ] qualifies as an implicit definition; we can consider it to be a legitimate meaning manual of the Cantorian set-concept and the membership-relation:

$$
U_{\mathrm{M}}(\text { set }, \in) \equiv\left\{\left\langle\mathcal{C}_{\mathrm{ZFC}}, \operatorname{AxZFC}[\text { set }, \in]\right\rangle\right\}
$$

where $\mathcal{C}_{\mathrm{ZFC}}$ stands for the mathematical context of doing Zermelo-Fraenkel set-theory.

\section{Exitum}

So far our argument has been in favour of the following conditional thesis: IF Horwich's Wittgensteinian theory of meaning (semantic deflationism) and our Criterion of implicit definability are adopted, THEN (i) we can define the set-concept and the membership-relation implicitly by means of a meaning manual which constitutes the meaning of the set-concept and the membership-relation; and THEN (ii) we can dissolve the conceptual perplexity of multitarian wholism by pointing out that the troublesome Expressions of 'seen as one object' and 'combined into a whole' were used in a context where they were never used before (they were used in other contexts, where their use did not evoke perplexity), i.e. their use manuals were not obeyed. Since we have only argued for the conditional and not for the condition, it seems we cannot endorse the consequent. Nevertheless we can.

Horwich [1998: 46-51] has argued in favour of semantic deflationism and we endorse his listed seven arguments wholeheartedly. Moreover, we claim that the present analysis of multitarian wholism ought to count as argument Number 8 in favour of semantic deflationism. For the power to solve or to dissolve philosophical problems that belong to the domain of the philosophical theory under consideration is reason enough to its acceptance. Since, as I have attempted to show (the if-then argument of this paper), semantic deflationism can deal with the conceptual problem of multitarian wholism successfully when enriched with our notion of implicit definability, this constitutes an abductive argument in favour of 
semantic deflationism. Salute Paul Horwich.

\section{ACKNOWLEDGMENTS.}

I am indebted to valuable comments of anonymous referees. These investigations were financially supported by the Dutch Organisation for Scientific Research (NWO), Section Philosophy and Theology.

F.A. Muller

Institute for History and Philosophy of Science and Mathematics

Utrecht University

P.O. Box 80.000, 3508 TA Utrecht, The Netherlands

f.a.muller@phys.uu.nl 


\section{References}

[Black 1971] M., 'The Elusiveness of Sets', Review of Metaphysics 24 (1971) 614-636

[Cantor 1895] G., 'Beiträge zur Begründung der transfinieten Mengenlehre I', Mathematische Annalen 46 (1895) 481-512

[Fraenkel 1973] Fraenkel, A.A., Bar-Hillel, Y., Lévy, A. and D. van Dalen, Foundations of Set Theory (2nd Ed.), Amsterdam: North-Holland, 1973

[Frege 1980] G., Philosophical and Mathematical Correspondence, (G. Gabriel, ed.), Oxford: Basil Blackwell, 1980

[Frege 1984] G., Collected Papers on Mathematics, Logic and Philosophy, (B. McGuiness, ed.), Oxford: Basil Blackwell, 1984

[Hale and Wright 2001] B. and C., 'Implicit Definition and the A Priori', in Reason's Proper Study. Essays towards a Neo-Fregean Philosophy of Mathematics, Oxford: Clarendon Press, 2001

[Hallett 1984] M., Cantorian Set Theory and Limitation of Size, Oxford: Clarendon Press, 1984

[Hilbert 1925] D., 'Über das Unendliche', Mathematische Annalen 95 (1925) 161-190

[Hodges 1993] W., Model Theory, Cambridge: Cambridge University Press, 1993

[Horwich 1998] P., Meaning, Oxford: Clarendon Press, 1998

[Jané 2001] I., 'Reflections on Skolem's Relativity of Set-Theoretical Concepts', Philosophia Mathematicae 9 (2001) 129-153

[Kanamori 2003] A., The Higher Infinite. Large Cardinals in Set-Theory from their Beginnings (2nd Ed.), Berlin: Springer-Verlag, 2003

[Lavine 1994] S., Understanding the Infinite, Cambridge, Massachusetts: Harvard University Press, 1994

[Neumann 1925] J. von, 'Eine Axiomatisierung der Mengenlehre', Journal für die Mathematik 154 (1925) 219-240, in: J. von Neumann, Collected Works I, A.H. Taub (ed.), Oxford: Pergamon Press, $1961,35-47$

[Muller, 2004] F.A., 'Deflating Skolem', to appear in Synthese

[Prior 1960] A.N., 'The Runabout Inference-Ticket', Analysis 21 (1960) 38-39

[Wang 1996] H., A Logical Journey. From Gödel to Philosophy, Cambridge, Massachusetts: MIT Press, 1996

[Whitehead \& Russell 1910] A.N. \& B.A.W., Principia Mathematica, Volume I, Cambridge: At the University Press, 1910

[Wittgenstein 1979] L., Cambridge Lectures 1932-1935 (A. Ambrose, ed.), Oxford: Basil Blackwell, 1979 